\title{
Donne autrici o vittime di reato? Un'indagine sull'efficacia delle misure alternative nei percorsi di recupero delle detenute nel contesto agrigentino
}

\author{
Ignazia Bartholini
}

\section{Riassunto}

Nel senso comune la pena detentiva è considerata un elemento inevitabile della società contemporanea. Essa continua ad essere percepita nell'immaginario collettivo come uno strumento di segregazione per coloro che sono riconosciuti colpevoli di particolari reati. I principi classici della prontezza, proporzionalità, infallibilità e dolcezza della pena non si identificano oggi esclusivamente con un edificio - la prigione - ma, sempre più di frequente, con strumenti alternativi e più efficaci per il recupero e la risocializzazione del soggetto. Proprio in questi decenni in cui l'intreccio fra povertà, penalità ed esclusione sociale diviene sempre più evidente, l'afflizione a cui il singolo è sottoposto coinvolge il sistema sociale e la sua inabilità nell'includere i soggetti più vulnerabili. Perciò, a quarant'anni dalla riforma penale, in un'ottica di reinserimento e risocializzazione, le più tradizionali risposte retributive sono sostituite da interventi di conciliazione e di tipo riparativo.

Il tema che viene analizzato riguarda l'efficacia delle misure alternative alla detenzione concesse alle donne condannate quale strumento di inclusione e di ridefinizione delle relazioni con la comunità, nel superamento dei limiti delle ossessioni securitarie che talvolta provengono da taluni ambienti sociali come quello - analizzato - del contesto agrigentino. Ciò ha presupposto, da un lato, una lettura del dettato normativo di riferimento e della letteratura sociologica concernente la criminalità femminile e, dall'altro, un'analisi complessiva della condizione femminile all'interno degli istituti di pena, attraverso l'analisi degli ultimi dati forniti dall'Istat sulla attuale situazione delle carceri. Per evidenziare, in ultimo, l'utilità delle misure alternative alla detenzione, è stata svolta una ricerca sulla loro efficacia attraverso l'analisi dei percorsi di recupero predisposti dall'U.E.P.E. di Agrigento per le donne detenute all'interno di un contesto sociale che ne fa delle vittime piuttosto che delle autrici consapevoli di reato. Tutto ciò ha consentito una valutazione, sia pure parziale per l'inevitabile limite prospettico che un case study porta con sé, dell'efficacia di tali misure.

\section{Résumé}

Au sens commun du terme, la peine de la détention est une caractéristique inévitable de la société contemporaine. Dans l'imaginaire collectif, elle continue d'être considérée comme un moyen pour isoler ceux qui ont été reconnus coupables de certains crimes. Aujourd'hui, les principes classiques de la rapidité, de la proportionnalité, de l'infaillibilité et de la douceur des peines ne s'identifient pas exclusivement à un bâtiment - la prison - mais, de plus en plus, aux mesures alternatives plus efficaces pour la réinsertion du condamné. C'est plus précisément au cours de ces dernières décennies que l'interaction entre la pauvreté, la pénalité et l'exclusion sociale devient de plus en plus évidente; par conséquent, l'affliction subie par l'individu affecte le système social et met en évidence ses difficultés d'intégrer les plus vulnérables. Pour cette raison, quarante ans après la réforme pénale, les réponses rétributives les plus classiques sont remplacées par des interventions de conciliation et réparatrices .

La question analysée par cet article concerne l'efficacité des mesures alternatives à la détention accordées aux femmes condamnées en tant que moyen d'intégration et de renouement des relations avec la communauté afin de dépasser les limites des obsessions sécuritaires qui proviennent parfois de certains milieux sociaux comme celui du territoire d'Agrigente qui a été analysé. Cela a supposé, d’un côté, une étude des normes et de la littérature sociologique concernant la criminalité des femmes; de l'autre, une analyse globale de la condition des femmes en prison par le biais des dernières données publiées par l'ISTAT (Institut National de la Statistique) sur la situation actuelle des institutions pénitentiaires en Italie.

Enfin, dans le but de montrer l'utilité des mesures alternatives à la détention, l'auteur a mené une recherche sur leur efficacité analysant les actions de réinsertion sociale mises en place par le U.E.P.E. (Service d'Insertion et de Probation) d'Agrigente au bénéfice des femmes détenues. Ces dernières, par rapport au contexte social d'origine, sont des victimes plutôt que des auteurs ayant consciemment commis les crimes.

Tout cela a permis à l'auteur d'effectuer une évaluation de l'efficacité de ces mesures, même s'il agit d'une évaluation partielle en raison des inévitables limites de perspective d'une étude de cas .

\footnotetext{
• Ricercatrice confermata, Dipartimento “Culture e società”, Università di Palermo.
} 


\begin{abstract}
In the common meaning of the term, incarceration is an unavoidable feature of contemporary society. In the collective imagination, it continues to be considered to be the way to isolate those who had been convicted of certain types of crime. Nowadays, classical principles of speed, proportionality, infallibility, and mildness of punishments do not exclusively identify a building - the prison - but increasingly identify also alternatives to detention which are the most effective forms of rehabilitation. Specifically in recent years, the interaction between poverty, penalty and social exclusion is becoming more and more evident; therefore, the affliction suffered by any individual affects all of society and highlights its problems in order to integrate the most vulnerable. For this reason, 40 years after penal reform, more traditional retributive sentencing practices are replaced by conciliation and restorative procedures.

This article deals with the issue of effectiveness of alternatives to incarceration granted to convicted women as a means to achieve social integration and meaningful relationships with the community in order to move beyond the limits of security obsessions that can be found in certain social environments such as the analysed province of Agrigento. This required, on the one hand, a study of laws and sociological literature regarding women's criminality; on the other, a global analysis of women prisoners' conditions using the latest data published by ISTAT (Italian Institute of Statistics) on the current situation in Italian penal institutions.

Finally, with the aim of demonstrating the usefulness of alternatives to detention, the author conducted research on their effectiveness by analysing social reintegration actions implemented by U.E.P.E. (Probation Service) of Agrigento for female prisoners. In relation to social context of origin, these women are victims rather than authors having consciously committed the crimes.

All this led the author to conduct an effectiveness evaluation of these measures, albeit a partial one due to inevitable limitations in a case-study.
\end{abstract}

Key words: women; alternatives to detention; prison; local contexts.

\section{Donne e crimine, una breve analisi} teorica.

Ciò che hanno cercano di spiegare gli studi recenti sulla criminalità femminile concerne per un verso il contributo di genere al manifestarsi della criminalità ${ }^{1}$, dall'altro il modo in cui essa si crea come prodotto sociale e il suo evolversi in funzione delle componenti culturali dell'ambiente di riferimento ${ }^{2}$. Il mondo della

${ }^{1}$ Burkhart K.W., Women in Prison, Double-day \& Co, New York, 1976; Walker H., "Women's issues in probation practice", in H. Walker \& B. Beamount (eds.), Working with offenders, Macmillan, London, 1985; de Cataldo Neuburger L., La criminalità femminile tra stereotipi culturali e malintese realtà, Cedam, Padova, 1996; Gelsthorpe L., “Female offending': a theoretical overview", in McIvor G. (ed.), "Women who offend". Research Highlights in Social Work 44, Jessica Kingsley Publishers, London, 2004.

2 Adler F., Sisters in Crime, McGraw- Hill Book Company New York, 1975; Buonanno R., L'altra donna. Devianza e criminalità, Adriatica, Bari, 1983; Faccioli F., I soggetti deboli. I giovani e le donne nel sistema penale, Franco Angeli, Milano, 1990; Feinman C., The Criminalization of a Woman's Body, Routledge, London, 1992; Pitch T., Donne in carcere. Ricerca sulla detenzione femminile in Italia, Feltrinelli, Milano, 1992; Hagan J., criminalità è stato frequentato ufficialmente, per gran parte e per lungo tempo, da uomini. Partendo dal presupposto che la criminalità femminile di livello "soft" (occasionale, minoritaria e disorganizzata), in opposizione a quella maschile di livello "hard" (organizzata e normalizzata), sia una costruzione sociale sedimentata nel tempo ${ }^{3}$, è possibile affermare che esistono delle rappresentazioni culturali in grado di orientare la percezione collettiva di ciò che è reato e di ciò che concerne i reati femminili nella loro caratterizzazione e valutazione. La costruzione sociale delle differenze che

Crime and Disrepute. Sociology of new Century, Pine Forge Press, Thousand Oaks (California), 1994; Naffine N., Feminism and Criminology, Cambridge Polity Press, Cambridge, 1997; Zucca M., Donne delinquenti. Storie di streghe, eretiche, ribelli, rivoltose, tarantolate, Edizioni Simone, Napoli, 2004; Hedderman C., Empty cells or empty words, Criminal Justice Alliance, London, 2012.

3 Carlen P., Worrall A., Analysing women's imprisonment, Willan, Cullompton, 2004; Chesney-Lind M., Irwin K., "Patriarchy, crime and justice: Feminist 
investono le identità maschili e femminili ha per lungo tempo riprodotto meccanismi di segregazione dei ruoli in virtù del fatto che la criminalità, come il carcere, tradizionalmente "sono domini maschili (...) mai esaminati come tali" 4 .

Molti studi recenti sottolineano quindi come la "marginalizzazione" della criminalità femminile sia il risultato di un dominio culturale maschile che pone in ombra la reale funzione delle donne all'interno del "topos" criminale, ma che non descrive adeguatamente il ruolo che di fatto esse hanno svolto ${ }^{5}$ Allo stesso modo la risocializzazione nei suoi strumenti legislativi e nelle sue pratiche solo di recente ha affrontato il tema delle discriminazioni di genere. Sono state soprattutto le donne ad occuparsi di questo tema ${ }^{6}$, ma il dibattito è ancora aperto e lungi dall'aver raggiunto un approdo concettuale.

Il legislatore, dal suo canto, appare quasi sempre come portatore di un atteggiamento indulgente e, quindi, segregante nei confronti delle donne. Nel porre in essere strumenti alternativi alla

criminology in era of backlash", Feminist Criminology, 1(1), 2008, pp. 6-26.

4 Pitch T., "Dove si vive, come si vive", in Campelli E., Faccioli F., Giordano V., Pitch T., Donne in carcere. Ricerca sulla detenzione femminile in Italia, Feltrinelli, Milano, 1992, pag. 26.

5 Edwards S., Gender, sex and law, Croom Helm, London, 1985; Minicucci G., Qui e altrove. Famiglie di Calabria e Argentina, Franco Angeli, Milano, 1989; Sibert R., Mafia e quotidianità, Il Saggiatore, Milano, 1996; Dino A. (in collaborazione con Principato T.), Mafia Donna. Le vestali del sacro e dell'onore, Flaccovio, Palermo, 1997; Dino A., Mutazioni. Etnografia del mondo di Cosa Nostra, La Zisa, Palermo, 2002; Dino A., Gli ultimi padrini. Indagine sul governo di Cosa Nostra, Laterza, Roma-Bari, 2011.

${ }^{6}$ Faccioli F., I soggetti deboli. I giovani e le donne nel sistema penale, Franco Angeli, Milano, 1990; Pitch T., Donne in carcere. Ricerca sulla detenzione femminile in Italia, Feltrinelli, Milano, 1992; Patel S., Stanley S., The use of the community order and the suspended sentence order for women, Center for Crime and Justice Studies, London, 2008. detenzione a loro rivolti, ha più volte sottolineato la necessità di evitare il gravitare nei circuiti penitenziari delle stesse, ma ciò "solo" in virtù della loro condizione di madri ${ }^{7}$ e non della persona stessa come autrice di reato.

Nel senso comune la pena detentiva è considerata un elemento inevitabile della società contemporanea. Essa continua, ancora oggi, ad essere percepita nell'immaginario collettivo come uno strumento di confinamento per coloro che sono riconosciuti colpevoli di particolari reati. Tuttavia, principi classici della prontezza, proporzionalità, infallibilità e dolcezza della pena non si identificano oggi esclusivamente con un edificio - la prigione - ma, sempre più di frequente, con strumenti alternativi e più efficaci al recupero e alla risocializzazione del soggetto. Il Consiglio d'Europa. dopo l'iniziale attenzione rivolta al sistema detentivo, già dagli anni '90, con le Raccomandazioni in materia di probation $\mathrm{R}(92) 16, \mathrm{R}(2000) 22$ e $\mathrm{R}(2010) 1$, ha spostato il proprio interesse nei confronti delle misure alternative alla detenzione, che vengono più propriamente definite community sanctions. Si tratta quindi di distinguere le funzioni sanzionatorie visibili, attraverso le mura del carcere, da quelle invisibili delle misure alternative, fatte di un reticolo immateriale di relazioni istituzionali ed umane, giuridiche e professionali che determina la realtà effettiva dell'ammesso alla misura. Proprio in questi decenni in cui l'intreccio fra povertà, penalità ed esclusione sociale diviene sempre più evidente, l'afflizione a cui il singolo è

\footnotetext{
7 Thomas C.W., "Prisonization or Resocialization? A Study of External Factors Associated with the Impact of Imprisonment", Journal of research in crime and Delinquency, 10(13), 1973, pp.13-21; Santoro E., Carcere e società liberale, Torino, Giappichelli, 2004; Mosconi
} 
sottoposto coinvolge il sistema sociale e la sua inabilità nell'includere i soggetti più vulnerabili. Perciò, a quarant'anni dalla riforma penale, in un'ottica di reiserimento e risocializzazione, le più tradizionali risposte retributive sono sostituite da interventi di conciliazione e di tipo riparativo. Le community sanctions non escludono un retablo di relazioni "necessarie" alla ricostruzione identitaria dell'ammesso alla misura. Si tratta quindi di analisi quantitative quelle che vengono sviluppate qui di seguito che si intrecciano con i prodromi di rappresentazioni sociali, di percezioni eteroriflesse, di relazioni significative necessarie ad un effettivo percorso di recupero delle detenute.

\section{Pene afflittive e misure alternative.}

La tabella n. 1 descrive efficacemente $\mathrm{i}$ numeri del "sovraffollamento" dei detenuti ospitati che superano, nel rapporto fra il numero degli istituti penitenziari e il totale della popolazione detenuta, la capienza regolamentare prevista tranne che in qualche caso (gli istituti penitenziari della Basilicata, Abruzzo, Piemonte, Sardegna, Toscana, Trentino-Alto Adige, Valle D'Aosta). È da precisare però che nel numero complessivo sono compresi anche coloro che si trovano in carcere in regime di semilibertà. Il numero dei detenuti stranieri è più alto nelle regioni centro-settentrionali rispetto a quelle meridionali, ad eccezione della Sicilia in cui, a fronte di 23 istituti penitenziari presenti, il numero totale dei detenuti, in buona parte stranieri, supera di poche decine la capienza regolamentare. Ai fini della nostra ricerca un posto a parte occupano le donne che, invece,

G., Sarzotti C., Antigone in carcere, Carocci Editore, sono presenti, come nelle restanti regioni, in una percentuale marginale e il loro numero rispetto al totale risulta piuttosto esiguo.

Nella situazione attuale il fenomeno della detenzione femminile deve essere ricondotto ai mutamenti sociali, politici ed economici che hanno interessato il nostro Paese $^{8}$ e che hanno visto insieme ai reati comunemente attribuiti alle donne - come la prostituzione - anche l'ingresso delle medesime nell'area dei reati di tipo mafioso e organizzati. Sono frequenti i reati legati alla tossicodipendenza (spaccio e detenzione di sostanze stupefacenti), in cui le donne straniere vengono utilizzate come "manovalanza" dalle organizzazioni criminali9, e i reati contro il patrimonio (furto, rapina, taccheggio, estorsione); infine solo una piccola percentuale è accusata di omicidio e generalmente si tratta di reazioni a violenze subite in prima persona o dai figli.

Tra gli aspetti considerati per spiegare la tipicità dei reati femminili vi è quella relativa al disagio sociale. La condizione di povertà sembra anche offrire una spiegazione ai tassi di recidiva.

Negli ultimi anni si è poi registrato un incremento della partecipazione femminile alla criminalità organizzata: è il caso di donne fedeli al contesto mafioso di appartenenza oppure, al

Roma, 2004.

8 Vitello F., Stefanizzi S., "Genere e criminalità: alcune riflessioni sui dati delle statistiche giudiziarie", in de Cataldo Neuburger L., La criminalità femminile tra stereotipi culturali e malintese realtà, Cedam, Padova, 1996, pag. 84.

${ }^{9}$ Faccioli F., "Le donne in carcere: la composizione sociale, i reati, le pene", in Campelli E. et al., Donne in carcere. Ricerca sulla detenzione femminile in Italia, Feltrinelli, Milano, 1992; Dino A. (in collaborazione con Principato T.), Mafia Donna. Le vestali del sacro e dell'onore, Flaccovio, Palermo, 1997; Dino A., Mutazioni. Etnografia del mondo di Cosa Nostra, La Zisa, Palermo, 2002. 
contrario, ribelli e collaboratrici di giustizia.

Si evidenzia inoltre l'alto tasso di presenza di donne straniere all'interno delle carceri: sono molto spesso madri separatesi dai figli lasciati nel loro paese di origine; sono donne che sentono maggiormente il peso della detenzione perché completamente sole e lasciate al loro destino; sono donne che generalmente non hanno altra scelta se non quella di delinquere per sopravvivere. A monte non c'è solo un problema linguistico, ma un vero e proprio conflitto culturale che le separa dalla società degli altri e le fa vivere in una solitudine devastante.

\section{Tratti caratterizzanti il proprium delle} donne detenute nelle leggi e nelle riforme.

\subsection{La Legge di riforma dell'Ordinamento}

\section{Penitenziario n. $354 / 75$.}

La riforma dell'Ordinamento Penitenziario Legge n. 354/75 - rappresenta la prima e significativa presa di distanza dalla pratica della detenzione stabilita dal Codice Rocco che si basava su: 1. una concezione del carcere come istituzione chiusa, con una propria gerarchia interna da rispettare; 2. l'isolamento dei detenuti all'interno del carcere, che porta ad una spersonalizzazione del detenuto (che viene chiamato col numero di matricola e non con il cognome); 3. la rigida separazione tra carcere e mondo esterno, con la limitazione alle pratiche religiose, lavoro e istruzione come uniche attività consentite.

La novità più importante introdotta dalla legge di riforma dell'Ordinamento Penitenziario - Norme sull'ordinamento penitenziario e sulla esecuzione delle misure privative e limitative della libertà - è quella contenuta al Capo VI che alla pena in carcere affianca quella delle misure alternative alla detenzione con cui, in casi particolari prescritti dalla norma, viene prevista la possibilità di scontare parte della pena fuori dal carcere, favorendo così il ritorno nell'ambiente sociale di appartenenza del condannato. Viene a tal fine prevista la figura del Magistrato di Sorveglianza (Capo II e III del Titolo II), incaricato di istruire le pratiche per il procedimento di concessione della misura alternativa, nonché i Centri di Servizio Sociale per Adulti (oggi divenuti Uffici di Esecuzione Penale Esterna), delegati dal Magistrato di Sorveglianza ad effettuare l'indagine socio-familiare necessaria per l'ottenimento della misura e ad espletare la funzione di controllo della stessa. Sembra essere messa in primo piano la figura del condannato allorquando, all'art. 13 della suddetta legge, si predispone che "il trattamento penitenziario deve rispondere ai particolari bisogni della personalità di ciascun soggetto", per consentire una "individualizzazione" della pena che meglio si adatti al suo percorso di risocializzazione.

Un primo accenno alle donne detenute è contenuto all'art. 21 bis della stessa legge $\mathrm{n}$. 354/75 e recita che "le condannate e le internate possono essere ammesse alla cura e all'assistenza all'esterno dei figli di età non superiore agli anni dieci", limitando in questo modo il problema della detenzione femminile allo status di "madre detenuta". A norma dell'art. 50 della stessa legge, sono ammessi al beneficio del regime di semilibertà tutti coloro che sono stati condannati ad una pena dell'arresto o della reclusione non superiore a sei mesi e tutti coloro che abbiano scontato metà di una pena più lunga. Elemento base per la concessione del beneficio deve 
comunque essere la volontà di reinserirsi nella vita sociale, attraverso uno specifico programma.

\subsection{Legge 66/1986 (Legge Gozzini) e successive modifiche.}

La legge n. 663 del 10 Ottobre 1986 - Modifiche alla legge sull'ordinamento penitenziario e sulla esecuzione delle misure privative e limitative della libertà - conosciuta comunemente come "legge Gozzini", si presenta come un'integrazione della legge 354/75. La norma, infatti, prevede una maggiore apertura dell'istituzione carceraria al mondo esterno dando la possibilità ai soggetti del Terzo Settore di partecipare al trattamento rieducativo. Prevede maggiori possibilità di decarcerizzazione del soggetto condannato, attraverso l'ampliamento di misure che consentono un'uscita temporanea dal carcere. Gli artt. 6 e 9 della legge recitano rispettivamente: "I detenuti e gli internati possono essere assegnati al lavoro all'esterno. Ai detenuti assegnati al lavoro extramurario è consentito di prestare la loro opera senza scorta, salvo che essa sia ritenuta necessaria per motivi di sicurezza. Gli imputati sono ammessi al lavoro all'esterno previa autorizzazione della competente autorità giudiziaria" e "Ai condannati che hanno tenuto regola condotta, e che non risultano di particolare pericolosità sociale, il Magistrato di Sorveglianza, sentito il direttore dell'istituto, può concedere permessi premio di durata non superiore ogni volta a quindici giorni per consentire di coltivare interessi affettivi, culturali o di lavoro". Viene quindi permesso un graduale reinserimento nella società del condannato, anche nei casi in cui debba scontare parte della sua pena. La legge, inoltre, prevede l'applicazione delle misure alternative alla detenzione anche per coloro che si trovano in regime di custodia cautelare al fine di consentirne la continuazione delle attività scolastiche o di tipo formativo e di aver cura della propria famiglia favorendo così la possibilità di rieducare un condannato nel suo stesso habitat socio-culturale.

Anche in questo testo normativo, l'unico accenno contenuto nei riguardi delle donne detenute è legato alla loro condizione di madre. L'art. 13, pur non focalizzandosi esclusivamente sulle donne detenute, quanto invece su soggetti diversamente vulnerabili, così recita: "La pena della reclusione non superiore a due anni, anche se costituente parte residua di maggior pena, nonché la pena dell'arresto, possono essere espiate, se non vi è stato affidamento in prova al servizio sociale, nella propria abitazione $\mathrm{o}$ in altro luogo di privata dimora ovvero in un luogo pubblico di cura o di assistenza quando trattasi di: 1) donna incinta o che allatta la propria prole ovvero madre di prole di età inferiore ai tre anni con lei convivente; 2) persona in condizioni di salute particolarmente gravi che richiedono costanti contatti con i presidi sanitari territoriali; 3) persona di età superiore a 65 anni, se inabile anche parzialmente; 4) persona di età minore a 21 anni, per comprovate esigenze di salute, di studio, di lavoro e di famiglia".

La Legge Gozzini è stata in seguito integrata in alcune sue parti dalla Legge del 27 maggio 1998 n. 165 - Modifiche all'articolo 656 del codice di procedura penale ed alla legge 26 Luglio 1975 n. 354 e successive modificazioni - conosciuta come "Legge Simeone-Saraceni”, che introduce la sospensione dell'esecuzione della pena detentiva, qualora non superiore a tre anni o a quattro nel caso di 
affidamento in prova in casi particolari, per consentire al condannato di presentare istanza per la concessione di una delle misure alternative alla detenzione. In questo modo favorisce l'ammissione alle misure evitando l'esperienza carceraria nel caso di pene brevi. Essa sembra "spezzare" il continuum legislativo creato dalle norme precedenti perché prescrive un uso delle misure alternative alla detenzione come un provvedimento "svuota carceri»" denaturalizzando il concetto rieducativo della pena.

In particolare, all'art. 4, la legge dispone la misura alternativa della detenzione domiciliare qualora "La pena della reclusione non superi $\mathrm{i}$ quattro anni, anche se costituisce residuo di maggior pena". A questo particolare regime alternativo è ammessa anche "la donna incinta o madre di prole di età inferiore ad anni dieci, con lei convivente".

Rispetto alla precedente legge Gozzini, la legge Simeone-Saraceni innalza il tetto degli anni di pena per ottenere la detenzione domiciliare da due a quattro e gli anni dei bambini che hanno diritto ad essere accuditi dalla madre convivente da tre a dieci. Essa tuttavia si limita a ampliare delle normative preesistenti, senza fornire apporti significativi in materia di detenzione femminile né, più in generale, sul piano della filosofa della pena e dell'alternativa alla detenzione.

\subsection{D.P.R. n. 230 del 2000: il nuovo Regolamento}

\section{Penitenziario.}

Con il D.P.R. n. 230 del 2000 - Regolamento recante norme sull'ordinamento penitenziario e sulle misure privative e limitative della libertà - viene adottato il nuovo regolamento di esecuzione dell'ordinamento penitenziario. Esso, all'art. 1, comma 1 e 2, afferma che "Il trattamento degli imputati sottoposti a misure privative della libertà consiste nell'offerta di interventi diretti a sostenere i loro interessi umani, culturali e professionali. Il trattamento rieducativo dei condannati e degli internati è diretto, inoltre, a promuovere un processo di modificazione delle condizioni e degli atteggiamenti personali, nonché delle relazioni familiari e sociali che sono di ostacolo ad una costruttiva partecipazione sociale". Viene sottolineata quindi la crucialità della promozione di un processo di revisione critica da parte del detenuto della propria condizione finalizzato ad un miglioramento della propria vita sociale. Viene inoltre evidenziata l'importanza del mantenimento dei contatti con la famiglia che deve avvenire in maniera continuata per garantire la serenità psico-fisica del condannato (attraverso colloqui, corrispondenza epistolare, telefonate mensili) e vengono favoriti $i$ percorsi di istruzione e formazione, qualora il soggetto ristretto ne dimostri attitudine e interesse, oltre che le attività lavorative. Inoltre, sempre con riferimento ai rapporti con il nucleo familiare, il regolamento, al comma 2 dell'art. 61 afferma che "Particolare attenzione è dedicata ad affrontare la crisi conseguente l'allontanamento del soggetto dal nucleo familiare, a rendere possibile il mantenimento di un valido rapporto con i figli, specie in età minore, e a preparare la famiglia, gli ambienti prossimi di vita e il soggetto al rientro nel contesto sociale", rivolgendosi non solo ai padri, ma anche alle madri ristrette, al fine di favorire la creazione di un legame affettivo con $i$ figli, anche se a distanza e ad intervalli di tempo. 


\subsection{La Legge n. 40/2001 (Legge Finocchiaro).}

In materia di donne detenute, o meglio di madri detenute, una delle leggi recenti più importanti è la legge n. 40 del 2001, Misure alternative alla detenzione a tutela del rapporto tra detenute e figli minori. Conosciuta come "legge Finocchiaro", all'art. 1, prevede la modifica dell'art. 146 del codice penale relativo al rinvio dell'esecuzione della pena che viene differita "se deve aver luogo nei confronti di donna incinta; se deve aver luogo nei confronti di madre di infante di età inferiore ad anni uno".

Al fine di evitare la detenzione di minori, la legge prevede la creazione di due nuovi istituti: la detenzione domiciliare speciale e l'assistenza all'esterno dei figli minori. Ai sensi dell'art. 3 della legge in questione "Quando non ricorrono le condizioni di cui all'art. 47-ter (della legge $354 / 75)$, le condannate madri di prole di età non superiore ad anni dieci, se non sussiste un concreto pericolo di commissione di ulteriori delitti e se vi è la possibilità di ripristinare la convivenza con i figli, possono essere ammesse ad espiare la pena nella propria abitazione, $o$ in altro luogo di privata dimora, ovvero in un luogo di cura, assistenza o accoglienza, al fine di provvedere alla cura e alla assistenza dei figli, dopo l'espiazione di almeno un terzo della pena ovvero dopo l'espiazione di almeno quindici anni nel caso di condanna all'ergastolo".

L'altro istituto delineato dalla legge Finocchiaro per la salvaguardia del legame madre detenutafiglio, riguarda l'assistenza all'esterno dei figli minori, art. 5, secondo cui: "Le condannate e le internate possono essere ammesse alla cura e all'assistenza all'esterno dei figli di età non superiore agli anni dieci, alle condizioni previste dall'art. 21. La misura dell'assistenza all'esterno può essere concessa, alle stesse condizioni, anche al padre detenuto, se la madre è deceduta o impossibilitata e non vi è modo di affidare la prole ad altri che al padre".

La legge, quindi, si propone di evitare la carcerazione di minori figli di una madre detenuta. Questi istituti sono nati non solo per garantire alla madre il mantenimento, altrimenti interrotto, delle relazioni con il figlio, ma anche e soprattutto per preservare il benessere psicofisico del minore.

$\mathrm{La}$ funzione di controllo e supporto nell'esecuzione delle misure è affidata agli assistenti sociali operanti nell'Esecuzione Penale Esterna.

Malgrado i buoni propositi, la suddetta legge presenta seri limiti: innanzitutto si rivolge alle sole donne con condanna definitiva; prevede, come requisito fondamentale, il possesso di un'abitazione che permetta la convivenza con i figli, il che esclude già a priori gran parte delle donne straniere; e, infine, prevede come limite la stessa possibilità di reiterare il reato, privando così dei benefici le donne che presentano un alto tasso di recidiva.

\subsection{La legge n. 62/2011.}

Emanata successivamente, la legge n. 62/2011 all'art. 13 recita che "La pena della reclusione non superiore a due anni, anche se costituente parte residua di maggior pena, nonché la pena dell'arresto, possono essere espiate, se non vi è stato affidamento in prova al servizio sociale, nella propria abitazione $\mathrm{o}$ in altro luogo di privata dimora ovvero in un luogo pubblico di cura o di assistenza quando trattasi di: 1) donna incinta o che allatta la propria prole ovvero madre di prole di età inferiore ai tre anni con lei 
convivente; 2) persona in condizioni di salute particolarmente gravi che richiedono costanti contatti con i presidi sanitari territoriali; 3) persona di età superiore a 65 anni, se inabile anche parzialmente; 4) persona di età minore a 21 anni, per comprovate esigenze di salute, di studio, di lavoro e di famiglia".

$\mathrm{Si}$ evidenzia, quindi, un dettato non esclusivamente indirizzato alle detenute madri, ma ad una serie di soggetti che vivono una situazione di disagio. Il testo normativo prevede anche, all'art. 2, la possibilità della madre o del padre di assistere, fuori dal carcere, il figlio che si trovi in una condizione di imminente pericolo di vita o in gravi condizioni di salute. Ma la novità più importante di questa legge riguarda il comma 3 dell'art. 1 che, al fine di permettere alle madri che non abbiamo una fissa dimora di usufruire dell'opportunità di espiare la pena fuori dal carcere, per favorire la crescita del proprio figlio, fornisce al giudice la possibilità "di disporne la custodia presso un istituto a custodia attenuata per detenute madri, ove le esigenze cautelari di eccezionale rilevanza lo consentano”.

Gli Istituti a Custodia Attenuata per le madri detenute sono strutture che rivoluzionano il concetto di restrizione della libertà perché le detenute che vi abitano insieme ai figli sono sottoposte alle stesse prescrizioni del carcere, malgrado ciò avvenga in un ambiente che non turba il naturale sviluppo del bambino. Pur mantenendo lo status di istituto penitenziario, questi Istituti sono costruiti come un'abitazione civile, non sono dotati di impianti di sicurezza visibili ai bambini e sono collegati in rete ai principali servizi socio-assistenziali e sanitari del territorio, per favorire un programma di reinserimento e recupero sociale mediante attività di istruzione, formazione e lavoro.

\subsection{La legge n. 67/2014.}

L'ultima legge n. 67/2014, promulgata in materia di misure alternative alla detenzione, applica, così come già avviene per i minori, anche agli adulti la sospensione del procedimento del condannato con la conseguente messa alla prova. L'art. 3 della legge prevede la possibilità di richiedere la misura alternativa con "la sola pena edittale pecuniaria o con la pena edittale detentiva non superiore nel massimo a quattro anni, sola, congiunta o alternativa alla pena pecuniaria, nonché per $i$ delitti indicati dal comma 2 dell'articolo 550 del codice di procedura penale". Condizione fondamentale per la sua concessione, è l'affidamento dell'imputato al Servizio Sociale che predispone un programma di attività nell'ambito del volontariato di rilievo sociale e la prestazione di un lavoro di pubblica utilità, che "consiste in una prestazione non retribuita, affidata tenendo conto anche delle specifiche professionalità ed attitudini lavorative dell'imputato, di durata non inferiore a dieci giorni, anche non continuativi, in favore della collettività, da svolgere presso lo Stato, le regioni, le province, i comuni, le aziende sanitarie o presso enti o organizzazioni, anche internazionali, che operano in Italia, di assistenza sociale, sanitaria e di volontariato" (Art. 3). L'esito positivo della misura estingue il reato, in caso contrario essa è revocata.

\section{L'altro carcere: misure alternative alla detenzione.}

4.1 Il beneficio, la sospensione, il debito.

Le misure alternative alla detenzione 
rappresentano l'insieme degli istituti denominati "l'altro carcere": istituiti dalla legge di riforma dell'Ordinamento Penitenziario (legge $n$. 354/1975) e successive modifiche, le misure alternative rappresentano appunto l'alternativa alla detenzione presso gli istituti di pena. Esse possono essere applicate ai soli soggetti condannati, coloro cioè per cui è stata emanata una sentenza definitiva di colpevolezza e per i quali è stata comminata una pena associata al reato commesso. Non tutte le misure alternative sono uguali, considerando l'esecuzione e la durata: l'unico elemento che le accomuna tutte è la finalità che consiste "non solo nell'evitare il carcere sostituendolo col controllo del delinquente in ambiente libero, ma soprattutto nel favorire il reinserimento del soggetto grazie all'opera di personale specializzato"10. Con riguardo al contenuto della misura, il beneficio prodotto è la sospensione della condanna e l'espiazione del debito sotto la supervisione dell'Ufficio di Esecuzione Penale Esterna (vedasi tabelle n. 2 e n. 3).

Le misure alternative alla detenzione hanno alla base la consapevolezza della convergenza fra i condizionamenti dell'ambiente circostante e le scelte individuali nella commissione del reato. Essendo la devianza un prodotto culturale e sociale, il reo non va segregato in un istituto di pena, ma va inserito in un programma trattamentale che focalizzi l'attenzione sulla sua personalità e punti al suo reinserimento sociale. Come afferma F. Leonardi, Direttore dell'Osservatorio delle misure alternative presso la Direzione Generale dell'esecuzione penale

${ }^{10}$ Breda R., Coppola C., Sabattini A., Il Servizio Sociale nel sistema penitenziario, Giappichelli Editore, Torino, 1999, pag. 49. esterna del Ministero della Giustizia, "la valutazione sulle attività trattamentali realizzate sui condannati in misura alternativa alla detenzione raggiunge la massima utilità se è finalizzata all'applicazione pratica delle conoscenze acquisite per ottenere la riduzione del fenomeno criminale" ${ }^{11}$. In tal senso i dati di cui disponiamo esplicitano un trend positivo concernente gli effetti della misura.

Gli alti tassi di recidiva dimostrano l'inutilità del trattamento penitenziario e quindi i fattori che scoraggiano la reiterazione del reato sono da ricercarsi in primis nella rete sociale di supporto esterna al carcere ${ }^{12}$. In ambito nazionale sono rari i rapporti di cooperazione tra istituti di pena e territorio, soprattutto nel momento del fine pena, quando il soggetto viene lasciato in balia di sé stesso. L'unica chance di riabilitarsi $\mathrm{e}$ gradualmente inserirsi nel contesto di appartenenza è costituita dalle misure alternative che, a seconda delle situazioni personali del soggetto, legate al possesso di un alloggio, ad un'opportunità lavorativa, alla presenza di un supporto familiare, decretano il ripristino delle normali condizioni di vita del soggetto nella quotidianità.

Nell'Ordinamento Penitenziario italiano le misure alternative alla detenzione sono l'affidamento in prova al Servizio Sociale, la detenzione domiciliare, la semilibertà, la liberazione anticipata.

\subsection{Affidamento in prova al Servizio Sociale.}

\footnotetext{
${ }^{11}$ Leonardi F., "Le misure alternative alla detenzione tra reinserimento sociale e abbattimento della recidiva", Rassegna di Studi penitenziari, n. 2, 2007, pag. 9.

12 Frudà L., Alternative al carcere. Percorsi, attori e reti sociali nell'esecuzione penale esterna: un approfondimento della ricerca applicata, Franco Angeli, Milano, 2006.
} 
L'affidamento in prova al Servizio Sociale è la misura più ampia prevista dalla riforma penitenziaria (art. 47). Questa misura è l'unica concepita come alternativa totale alla detenzione, per cui il soggetto che usufruisce del beneficio sconterà in affidamento un periodo uguale alla pena comminata, purché al reato a cui sia associata una pena detentiva superiore ai tre anni. Nella formulazione originaria della legge, per poter fare richiesta per la concessione della misura, il soggetto deve essere sottoposto all'osservazione scientifica della personalità da parte dell'équipe di riferimento dell'sstituto per almeno un mese, con lo scopo di verificare le caratteristiche psico-fisiche, educative e sociali del condannato/a. La competenza della concessione della misura alternativa spetta al Tribunale di Sorveglianza su cui ricade la giurisdizione dell'istituto di pena in cui il soggetto è ristretto e che, in caso di esito positivo, stabilisce le prescrizioni che devono essere rispettate, prima fra tutte l'affidamento, appunto, del soggetto all'Ufficio di Esecuzione Penale Esterna con l'obbligo di mantenere rapporti con codesto ufficio. Altre prescrizioni sono il reperimento di una dimora, lo svolgimento di un'attività lavorativa e/o di un'attività di volontariato, il divieto di frequentazione di certi locali e contesti ritenuti inidonei ${ }^{13}$. Preposto alla funzione di controllo è l'Ufficio di Esecuzione Penale Esterna, incaricato di scrivere periodicamente al Magistrato di Sorveglianza sugli sviluppi della misura. Altra funzione dell'U.E.P.E. è quella di aiuto, di sostegno del soggetto in misura

${ }^{13}$ Di Cara M., Gervasoni A., Steiner M.A., Riforma penitenziaria e intervento sociale, La Nuova Italia Scientifica, Roma, 1990. alternativa durante tutto il percorso. Nel caso in cui le prescrizioni non vengano rispettate, il Magistrato di Sorveglianza può predisporre la sospensione o revoca della misura, trasformandola in altre misure alternative $\mathrm{o}$ traducendo il resto della pena in detenzione da scontare in carcere. A norma del comma 12 dell'art. 47 della legge 354/75, "l'esito positivo del periodo di prova estingue il reato e ogni altro affetto penale".

La legge delinea anche il profilo di quello che è stato definito l'affidamento in prova al Servizio Sociale in casi particolari. Per "casi particolari" si intendono i soggetti affetti da dipendenza da alcol o da sostanze, cha stiano svolgendo un programma di recupero o che intendano cominciarlo. Le differenze con l'affidamento ordinario dunque sono da rintracciare sia nella tipologia dei destinatari (qui sono tossicodipendenti e alcoldipendenti) e nella presenza obbligatoria di un programma di recupero, iniziato o comunque programmato, presso un servizio pubblico per le tossicodipendenze (Ser'T) oppure presso una struttura privata (generalmente una comunità di recupero). Per evitare delle strumentalizzazioni della misura da parte del soggetto richiedente, il Tribunale di Sorveglianza, ai sensi del comma 4 art. 12 della legge 663/1986, "può acquisire copia degli atti di procedimento e disporre gli opportuni accertamenti in ordine al programma terapeutico concordato, e deve accertare che lo stato di tossicodipendenza o alcool-dipendenza o l'esecuzione del programma di recupero non siano preordinati al conseguimento del beneficio". Per questo motivo, il Magistrato di Sorveglianza può prendere visione del programma eventualmente concordato 
dall'utente e dal servizio e può richiederne degli aggiustamenti. Nel caso in cui il Magistrato di Sorveglianza si accerta dell'impossibilità di concedere la misura (che ricordiamo rappresenta un diritto del soggetto dipendente da alcol o da sostanze) può procedere con la concessione dell'affidamento ordinario. Anche in questo caso restano le prescrizioni di controllo sull'andamento della misura da parte degli assistenti sociali dell'U.E.P.E.

\subsection{La detenzione domiciliare.}

L'istituto della detenzione domiciliare è disciplinato dall'art. 47-ter della legge $\mathrm{n}$. 354/1975. È una misura che somiglia molto alla misura cautelare degli arresti domiciliari. Rispetto all'affidamento in prova al Servizio Sociale, la misura della detenzione domiciliare è più restrittiva e non contiene indicazioni trattamentali per un percorso rieducativo. $\mathrm{Si}$ tratta di scontare la pena presso la propria abitazione quando quest'ultima non sia superiore ai quattro anni, anche se parte residua di una pena superiore. Possono usufruirne i seguenti soggetti:

1. donna gravida o madre di prole di età inferiore ai dieci anni;

2. padre, esercente la potestà, di prole di età inferiore ad anni dieci con lui convivente, quando la madre sia deceduta o assolutamente impossibilitata a dare assistenza alla prole;

3. persona in condizioni di salute gravi, che richiedano un contatto continuo con i presidi sanitari;

4. ultra sessantacinquenne inabile, anche se parzialmente;

5. infraventunenne con comprovate esigenze di studio, salute, lavoro, famiglia (Art. 47-ter, comma 1, legge 354/75).

Non possono essere ammessi al beneficio coloro che detengono un collegamento con la criminalità organizzata ancora al momento del giudizio. La detenzione domiciliare viene sempre concessa per l'espiazione di pena detentiva non superiore ai due anni indipendentemente dalle prescrizioni di cui sopra, quando non può essere concesso l'affidamento in prova al Servizio Sociale. Deputato alla concessione della misura è il Tribunale di Sorveglianza; il Magistrato di Sorveglianza è deputato invece all'applicazione della misura, apportando le opportune modifiche; il controllo invece è lasciato agli organi di polizia giudiziaria. La misura può essere revocata se la persona non rispetta le prescrizioni, la più importante delle quali è non allontanarsi dalla propria abitazione; in caso contrario il soggetto verrà accusato di evasione.

La legge di riforma, all'art. 47-quinquies, disciplina la detenzione domiciliare speciale, in seguito modificata ed integrata dalla legge Finocchiaro n. 40 del 2001, "Misure alternative alla detenzione a tutela del rapporto tra detenute e figli minori’. La misura è riservata alle detenute madri di prole di età non superiore ad anni dieci con loro conviventi, che, secondo il comma 1 dell'art., "possono espiare la pena presso il proprio domicilio, $\mathrm{o}$ in una struttura pubblica $\mathrm{o}$ privata o in altro luogo deputato all'accoglienza e all'assistenza, dopo espiazione di almeno un terso della pena o di almeno quindici anni nel caso di condanna all'ergastolo". Anche per questa particolare forma valgono le stesse prescrizioni della forma di detenzione ordinaria. 


\subsection{La Semilibertà.}

Il regime di semilibertà viene inserito nel nostro ordinamento tra le misure alternative, anche se non è proprio una di quelle. Il soggetto sottoposto a tale regime trascorre metà della sua giornata in carcere e alcune ore fuori dall'istituto per svolgere un'attività lavorativa, di studio $\mathrm{o}$ comunque adatta al reinserimento sociale. A norma dell'art. 50 della legge 354/75, sono ammessi a questa forma di beneficio tutti coloro che hanno sono stati condannati ad una pena dell'arresto o della reclusione non superiore a sei mesi e tutti coloro che abbiano scontato metà di una pena più lunga. Elemento base per la concessione del beneficio deve comunque essere la volontà a reinserirsi nella vita sociale, attraverso un programma valido. Il regime di semilibertà è concesso dal Tribunale di Sorveglianza, su proposta del direttore dellistituto di pena o del soggetto interessato. Il direttore dell'istituto è l'unico responsabile della misura e dopo la sua concessione stila il programma trattamentale in cui è indicata l'attività che l'individuo dovrà svolgere nelle ore che trascorre fuori dal carcere e le prescrizioni da seguire anche all'interno dell'istituto carcerario. Il compito di assistenza e controllo resta all'Ufficio esecuzione Penale Esterna.

Nel caso in cui il soggetto si dimostri incapace di seguire le prescrizioni del programma o vengono meno alcune condizioni fondamentali (ad esempio perde il lavoro), la misura verrà revocata e il soggetto tornerà a scontare la pena interamente in carcere.

Per quanto riguarda l'aspetto trattamentale, la misura centra in pieno quello che è il principio dell'individualizzazione della pena, attraverso un programma stilato insieme al soggetto sulla base delle sue risorse personali e di quelle del suo ambiente di vita.

\subsection{Libertà vigilata, liberazione anticipata e remissione del debito.}

La libertà vigilata non è una vera e propria misura alternativa, ma è sempre un beneficio che viene concesso al condannato di espiare parte della sua pena in libertà, sotto il controllo e l'ausilio rispettivamente della Polizia Giudiziaria e dell'Ufficio di esecuzione Penale esterna, che deve riferire periodicamente al Magistrato di Sorveglianza circa l'andamento del beneficio.

La liberazione anticipata, nella sua formulazione originaria, consiste in una riduzione di pena, pari a 45 giorni per ogni semestre di pena scontato, che anticipa, appunto, la conclusione della detenzione. Essa è un diritto per il condannato che sconta una pena e può essere concessa sia al soggetto detenuto in carcere, sia al soggetto che sta scontando una misura alternativa. Requisito essenziale è l'attiva partecipazione al trattamento rieducativo. In questo senso, la liberazione anticipata potrebbe essere un incentivo a partecipare alle attività di trattamento, sia all'interno dell'istituto penitenziario sia in misura alternativa.

Altra misura prevista dal nostro ordinamento è quella della remissione del debito, che spetta a coloro che si trovano in delicate condizioni economiche e che abbiamo tenuto una regola condotta. ¿̀ concesso dal Magistrato di Sorveglianza e cancella il debito per le spese processuali e di mantenimento della persona condannata o internata. La remissione del debito rappresenta un'agevolazione al soggetto per evitare di gravare sulla sua situazione al momento del reinserimento in società. 


\section{La ricerca: indagine sulle ammesse a} misura alternativa nel contesto agrigentino.

La ricerca descritta in questa sede si è svolta per tutto fra il 2014 e i primi mesi del 2015, articolandosi in due fasi:

a. la prima, ha riguardato l'individuazione delle cartelle relative alle donne in misura alternativa alla detenzione avuti in carico dall'U.E.P.E. di Agrigento. Mediante il sistema informatico Pegaso sono stati individuati i fascicoli inerenti i casi da inserire nel campione di riferimento.

b. la seconda ha riguardato la rilevazione delle variabili da utilizzare per la ricerca attraverso la consultazione dei fascicoli delle utenti, riposti nell'archivio dell'ufficio, e delle relazioni (sociali e di sintesi), redatte dagli assistenti sociali incaricati.

Per quanto riguarda il campione, si è scelto di analizzare le informazioni concernenti le donne seguite nell'arco temporale compreso tra gli anni 2009-2014 che rientravano nelle seguenti categorie

- $\quad$ donne condannate ammesse alla misura alternativa dell'affidamento in prova al Servizio Sociale;

- donne condannate ammesse alla misura alternativa dell'affidamento in prova al Servizio Sociale in casi particolari;

- donne condannate ammesse alla misura alternativa della semilibertà;

- donne condannate ammesse alla misura alternativa della detenzione domiciliare;

- donne sottoposte alla misura di sicurezza non detentiva della libertà vigilata;

- $\quad$ donne detenute con condanna compresa nei tre anni sottoposte a osservazione scientifica della personalità.

I casi presi in esame, che corrispondono alla totalità del campione, sono stati in tutto 101. La tabella n. 4 descrive la distribuzione delle misure alternative concesse alle 101 donne del campione di riferimento:

- $\quad 39$ casi di ammesse alla "osservazione detenute"

- 44 casi di ammesse alla "detenzione domiciliare"

- 17 casi di ammesse all"'affidamento in prova al servizio sociale"

- 1 caso di assistenza domiciliare.

Per quanto riguarda i 39 casi di donne sottoposte all'osservazione scientifica della personalità è stata effettuata una scrematura preliminare eliminando dal campione coloro che avessero ottenuto una pena superiore ai tre anni. Delle 39 donne, 23 hanno terminato la loro pena in carcere; 11 hanno ottenuto la misura alternativa della detenzione domiciliare; 1 la detenzione domiciliare provvisoria; 3 sono state affidate in prova al Servizio Sociale e 1 ha ottenuto l'affidamento in prova al Servizio Sociale in casi particolari (perché tossicodipendente).

La maggior parte di queste donne sottoposte all'osservazione scientifica della personalità proveniva da altre case circondariali/case di reclusione di Italia, trasferite ad Agrigento per motivi disciplinari o altro; per cui una volta terminato il periodo di osservazione e preso visione della decisione del Magistrato di Sorveglianza il caso è stato "affidato" all'Ufficio di Esecuzione Penale Esterna competente per territorio.

Sia le donne a cui è stata concessa la misura 
alternativa della detenzione domiciliare e che compongono la totalità del campione (44 donne), che le 17 donne affidate in prova al Servizio Sociale sono tutte residenti/domiciliate nei vari comuni della provincia di Agrigento e, ricadendo sotto la competenza della stessa U.E.P.E. di Agrigento, sono state seguite dagli assistenti sociali incaricati per territorio.

Il campione preso in esame è stato analizzato attraverso la rilevazione delle seguenti variabili:

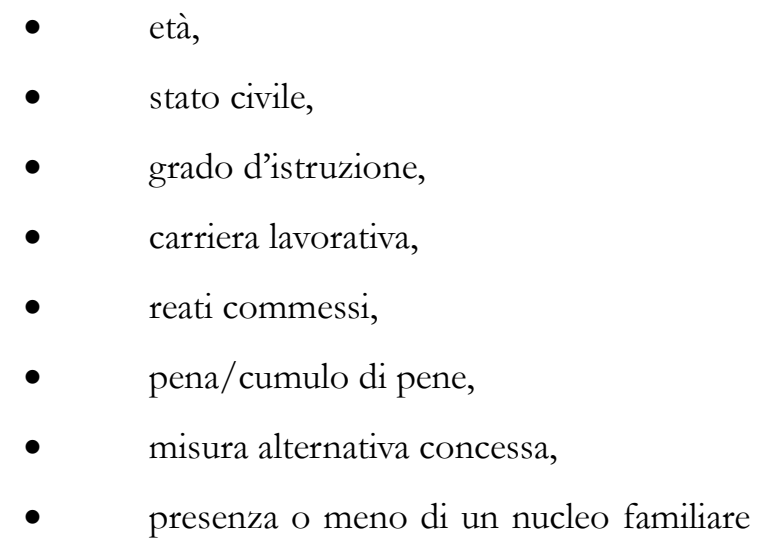

- $\quad$ presenza di eventuale recidiva.

Successivamente alla rilevazione dei dati, le variabili sono state analizzate singolarmente in modo da evidenziarne la distribuzione (vedasi tabella n. 5).

Dall'analisi effettuata si evidenzia inoltre che il $31,6 \%$ delle utenti ha un'età relativamente giovane e compresa tra i $31-40$ anni; il $42,5 \%$ ha conseguito la licenza media; il 42,5\% (la maggioranza) è coniugata; il $78,2 \%$ ha più di un figlio e, con riguardo all'occupazione, il 27,7\% delle donne del campione esaminato era casalinga al momento della condanna. La pena comminata a seguito di reato per il 50,5\% (la metà più uno) del campione di riferimento è stata inferiore ad un anno (tendenzialmente tra 1-6 mesi).
Per ciò che concerne i reati, dalla tabella n.6 si evidenzia come quello più usuale fra le donne dell'Agrigentino sia l'abusivismo edilizio, reato piuttosto frequente proprio in quell'area. In tale contesto, infatti, la socializzazione ha prodotto disposizioni e atteggiamenti che hanno per lungo tempo derubricato il reato di abusivismo edilizio a semplice furbizia ed applicazione di un “calcolo razionale dei vincoli e delle opportunità per il perseguimento di uno scopo (che ha) come causa remota la cultura e $\mathrm{i}$ processi di socializzazione" ${ }^{14}$. 亡̀ da considerare inoltre come le donne che entrano in contatto con l'esecuzione penale esterna lo sono spesso perché titolari dell'immobile a cui non è stata applicata una sanatoria attesa, ma soprattutto come front women di logiche devianti di tipo maschile basate su un scarso rispetto delle regole.

Come afferma Gucciardo, in contesti come il Sud d'Italia dove è assente o quasi assente l'autorità dello Stato che riesce a governare gli scambi sociali tra gli individui, "prevale il codice della furbizia, del calcolo costi/benefici, della massimizzazione del proprio benessere contro tutto e tutti”"15.

Dalla lettura dei dati si è registrato un elemento interessante: la metà delle donne condannate per abusivismo edilizio appartiene alla zona di Palma di Montechiaro, dove evidentemente esiste una "forma mentis" volta alla massimizzazione dei benefici a discapito della legge. D’altronde “un attore che valuta $\mathrm{i}$ costi e benefici, troverà razionale violare la legge e costruire

14 Gucciardo G., "Familismo amorale, modelli culturali e azione razionale", in Bartholini I. (a cura di), Capitale sociale, reti comunicative e culture di partecipazione, Franco Angeli, Milano, 2008, pag. 149.

15 Ibidem, pag. 148. 
abusivamente"16. Ne è prova il fatto che quasi tutte queste donne non sono consapevoli dell'illecito commesso, anzi loro si giustificano attribruendo la responsabilità ad un sistema che funziona male (tutti costruiscono così); proprio per questo generalmente la misura alternativa concessa è la detenzione domiciliare (dalla libertà) perché le pene comminate per il reato hanno durata di alcuni mesi associata a sanzioni amministrative elevate.

Immediatamente successivi per frequenza al reato di abusivismo edilizio sono quelli contro il patrimonio (furto, rapina, estorsione, ricettazione, ecc.): anche qui la maggior parte delle autrici di reato sembra essere spinta dal contesto di appartenenza generalmente povero di opportunità lavorative per le donne, soprattutto se straniere. L'elevato numero di questi reati sembra avvalorare la teoria della "marginalizzazione economica" di Naffine17 e Feinman ${ }^{18}$ : dove non esistono opportunità legittime per le donne, queste utilizzano le opportunità illegittime e delinquono.

Andando ad analizzare invece i reati legati alla detenzione e allo spaccio di sostanze stupefacenti, le donne che si macchiano di questo reato sono generalmente giovani, con una famiglia disgregata alle spalle e con reati dello stesso tipo; il reato viene commesso, nella maggior parte dei casi, in concorso con il compagno/marito perché entrambi tossicodipendenti. Inoltre molte di queste donne hanno ammesso di aver commesso altri reati perché sotto effetto di sostanze stupefacenti. In

${ }^{16}$ Ibidem, pag. 151.

17 Naffine N., Feminism and Criminology, Cambridge Polity Press, Cambridge, 1997.

${ }^{18}$ Feinman C., The Criminalization of a Woman's Body, Routledge, London, 1992. tutti i casi di donne tossicodipendenti si nota l'intervento del Servizio per le Tossicodipendenze (Ser.T.) in quanto esiste una volontà di sottoporsi al trattamento riabilitativo che non sempre, però, volge a buon fine.

Per quanto riguarda gli altri reati, essi si presentano in una percentuale minore, ma non irrilevante e sono essenzialmente legati a percorsi di disadattamento ed emarginazione sociale.

Riguardo al tasso di recidiva, la grande maggioranza si trova a commettere il primo reato e generalmente la misura alternativa funziona come deterrente speciale, soprattutto per coloro che non hanno mai gravitato nel circuito penitenziario e che quindi non conoscono le rigide regole che la detenzione comporta. Le donne sono maggiormente accondiscendenti alle prescrizioni del Magistrato di Sorveglianza e collaborano con gli operatori dell'ufficio esecuzione penale esterna rispetto agli uomini perché non vogliono perdere i benefici di cui godono o possono godere, soprattutto se madri. Tendenzialmente non reiterano il reato anche perché nel commetterlo sono state costrette da altri o da circostanze avverse. In generale, dunque, la concessione della misura alternativa sembra influire positivamente sui tassi di recidiva, almeno per le donne prese in esame.

Altro dato interessante emerso dalla ricerca risulta essere quello relativo alla famiglia, sia essa d'origine o acquisita: in generale risulta essere positivo, anzi "la qualità dei rapporti familiari sembra costituire una risorsa molto importante nell'esperienza della detenzione e dell'esecuzione 
penale esterna"19. Da una parte, la famiglia si rivela un'àncora per queste donne a cui appoggiarsi per superare il difficile momento; dall'altra, la famiglia si rivela un rifugio sicuro, lontano dagli sguardi giudicatori della gente, pronta a coprire e a cancellare quasi la vergogna e l'eventuale rifiuto sociale a seguito di un reato: in questo è possibile rintracciare gli atteggiamenti tipici di "familismo amorale"20 basantisi sulla propensione a cooperare all'interno della famiglia e sulla scarsa fiducia nelle istituzioni e nella società più in generale.

La stragrande maggioranza delle donne prese in esame è poi madre $(78,2 \%)$ e in questo caso la famiglia si rivela un supporto nell'accoglienza e nella crescita dei nipoti in modo da non interrompere il legame con il proprio habitat familiare. Dove questo non è possibile interviene l'istituzionalizzazione in case-famiglia, comunità $\mathrm{e}$ in casi estremi si ricorre all'affidamento familiare e all'adozione.

Rispetto alla famiglia d'origine, sembra essere meno presente la famiglia acquisita, da una parte perché generalmente anche il compagno o il marito è detenuto (per lo stesso reato o per altri reati), dall'altra perché i figli vengono spesso allontanati dal contesto familiare $O$, se maggiorenni, perché vivono in una propria famiglia.

La situazione si complica per le donne straniere che, nel campione di riferimento, risultano essere 11 su 101 casi presi in esame: in questo caso le donne non solo hanno delle difficoltà

\footnotetext{
${ }^{19}$ Campelli E., "Una risorsa ambivalente ed incerta: la famiglia", in Campelli E. et al. (a cura di), Donne in carcere. Ricerca sulla detenzione femminile in Italia, Feltrinelli, Milano, 1992, pag. 104.
}

comunicative e relazionali (soprattutto con le assistenti sociali la cui funzione viene spesso demonizzata o denigrata), ma non possono contare nemmeno su una famiglia in grado di supportarle; di conseguenza, generalmente, esse non riescono a godere dei benefici più ampi perché mancano i requisiti minimi del domicilio stabile e del lavoro. Una differenza significativa tra straniere e autoctone si evidenzia anche per quanto riguarda la tipologia di reato: le native, come si è già avuto modo di notare, sono accusate in maggioranza per il reato di abusivismo edilizio, forse perché legato ad una condizione socio-economica che permette alla donna di possedere un casa e ad un modello culturale che fa della costruzione abusiva una "regola contro le regole" accettabile e diffusa. Le donne straniere scontano pene per reati legati allo sfruttamento della prostituzione e per furto $\mathrm{o}$, in misura minore, per reati legati alla detenzione e allo spaccio di sostanze stupefacenti. Anche qui la tipologia dell'illecito commesso riflette una condizione di deprivazione economica e sociale, in quanto queste donne si trovano nel nostro contesto sole, lasciate in balia di loro stesse e delle condizioni del territorio che non lascia altra scelta se non delinquere per poter sopravvivere. Generalmente hanno figli che, a causa della lontananza della famiglia, vengono istituzionalizzati in case famiglia o affidati ad altre famiglie. La mancanza dei requisiti richiesti dal Legislatore (una casa, un'attività lavorativa) spesso non permette loro di godere di benefici alternativi o di misure più ampie rispetto a quelle concesse.

\footnotetext{
${ }^{20}$ Banfield E.C., The moral Basis of Blachward Society, The Free Press, Glencoe, 1958. (trad. it. Le basi morali di una società arretrata, il Mulino, Bologna, 2006).
} 
Si rileva, ad una lettura più approfondita delle cartelle esaminate, una certa ripetitività dei percorsi di vita delle donne ammesse alla misura e facenti parte del contesto socio-culturale agrigentino, uno stesso "iter della devianza" in molte delle storie raccontate da queste donne: si tratta generalmente di famiglie patriarcali in cui a lavorare è il padre, che si fa carico di tutta la famiglia e in cui la madre assume il ruolo di custode della famiglia, ma anche di "vittima sacrificale" spesso ignara del reato che le viene "consegnato" dalla propria famiglia. Inoltre, fino a pochi anni fa, era in uso la cosiddetta "fuitina", una forma impropria di ribellione all'autorità maschile che si traduce in una riaffermazione del "potere degli uomini" ed in una re-inclusione delle donne nel loro ruolo di madri e di mogli attraverso il matrimonio riparatore. Una riaffermazione quindi del patriarcato, prova ne è il fatto che molte di queste donne hanno abbandonato gli studi in concomitanza del matrimonio. Questa stessa "sorte" le accomuna alle donne relativamente più "anziane" del campione esaminato.

Donne di un contesto sociale - quello agrigentino - che sembra ostinato a rimanere ancorato al passato patriarcale e familista anche e soprattutto in contesti devianti o criminali.

\section{Riflessioni conclusive.}

Il sistema italiano nell'ultimo cinquantennio si è trasformato da un sistema meramente detentivo ad uno duplice - detentivo-alternativo - che tuttavia non sempre è riuscito a raggiungere gli obiettivi del reinserimento e della risocializzazione degli ammessi alla misura. Il quadro normativo italiano delinea, come descritto in precedenza, tutta una serie di misure alternative alla detenzione in grado di sottrarre il soggetto, che si presenti particolarmente meritevole degli stessi, al circuito penitenziario, ma ciò che è ancora latente e/o insufficiente è l'accompagnamento di coloro che hanno concluso il loro percorso nei circuiti penali.

Ciò nonostante, scontare la pena in misura alternativa alla detenzione riduce comunque il rischio di recidiva rispetto all'esecuzione penale in carcere: le misure alternative interrompono, spesso, gli effetti negativi prodotti dal sistema detentivo e facilitano percorsi di reinserimento attivo, sia pure pro tempore, nel tessuto sociale. Quanto emerge dalle osservazioni dimostra chiaramente gli effetti positivi delle misure alternative, con riferimento alla ricaduta nella recidiva, riportando valori analoghi a quelli riscontrati sulla totalità dei casi osservati, assicurando il buon esito di tali strumenti in termini sia di sanzione, sia di controllo sociale anche nel lungo periodo e, dunque, di prevenzione ${ }^{21}$.

Tuttavia è anche da considerare come i contesti sociali si diversificano per una serie di indicatori e che, nel caso specifico del contesto locale su cui abbiamo definito gli indicatori sopradescritti, si evidenzia la necessità di una risocializzazione che si ponga anche in dinamiche decontestualizzate, che definiscano una qualche distanza con una Lebenswelt di "cattivo" capitale sociale. Come ho avuto modo di scrivere qualche anno fa, "ogni contesto di riferimento è una piccola società, cioè un sistema composto dai sottosistemi di Stato, mercato, TS e famiglie. Ognuno di questi sottosistemi utilizza un mezzo

${ }^{21}$ Leonardi F., "Le misure alternative alla detenzione tra reinserimento sociale e abbattimento della 
simbolico di interscambio specifico rispettivamente: potere, denaro, reciprocità e dono - e genera beni di tipo peculiare rispettivamente beni pubblici, beni privati, beni relazionali secondari, beni relazionali primari" 22 . In un sistema, come quello meridionale, le forme di legalità sono spesso "deboli" e si sviluppano tali proprio in previsione del fatto che le norme pubbliche vigenti saranno frequentemente inefficaci, applicate in modo distorto e quindi inaffidabili ed eludibili. In opposizione al sedimentato socio-culturale, la criminalità femminile agrigentina non può considerarsi "soft $"$ - occasionale, minoritaria e disorganizzata ${ }^{23}$ - ma, al contrario, di livello “hard" (organizzata e normalizzata) per affiliazione. Essa può a buon diritto essere considerata come il prodotto di "relazioni capitalizzate" che conservano un certo grado di persuasività in rapporto alle componenti culturali dell'ambiente di riferimento quando le istituzioni pubbliche sono, in questo senso, particolarmente deboli $^{24}$.

Ciò pone il problema dell'effettiva efficacia delle

recidiva”, Rassegna di Studi penitenziari, n. 2, 2007, pp. 7-26.

22 Bartholini I., "Introduzione", in Id. (a cura di), Capitale sociale, reti comunicative e culture di partecipazione, Franco Angeli, Milano, 2008, pag. 26.

${ }^{23}$ Carlen P., Worrall A., Analysing women's imprisonment, Willan, Cullompton, 2004; Chesney-Lind M., Irwin K., "Patriarchy, crime and justice: Feminist criminology in era of backlash", Feminist Criminology, 1(1), 2008, pp. 6-26.

${ }^{24}$ Feinman C., The Criminalization of a Woman's Body, Routledge, London, 1992; Pitch T., Donne in carcere. Ricerca sulla detenzione femminile in Italia, Feltrinelli, Milano, 1992; Hagan J., Crime and Disrepute. Sociology of new Century, Pine Forge Press, Thousand Oaks (California), 1994; Naffine N., Feminism and Criminology, Cambridge Polity Press, Cambridge, 1997; Hedderman C., Empty cells or empty words, Criminal Justice Alliance, London, 2012; Chesney-Lind M., Irwin K., "Patriarchy, crime and justice: Feminist misure alternative nel loro specifico mandato risocializzante. Infatti, "È una scelta operativa fondata sulla 'fiducia' che il condannato risulti capace di 'recuperare il controllo del proprio comportamento', utilizzando un sistema di interventi che ha natura diversa da quelle repressivi tradizionali, favorendo e sostenendo l'assunzione di atteggiamenti di responsabilità e di autodeterminazione nell'esecuzione della misura"25. La "forza del legame sociale" che è alla base della messa alla prova, e che costituisce una pratica di ricostituzione del legame fra il detenuto e il mondo extramurario, deve quindi essere analizzata alla radice dagli operatori perché, qualora il contesto consideri la pratica del reato come la "normalità dell'esistenza", è facile supporre che le possibilità di riduzione della recidiva si assottiglino. $\mathrm{E}$ ciò in contesti, ancora viziati dal patriarcalismo, che ostinatamente considerano le mogli e le figlie di criminali o di soggetti genericamente devianti, nella strumentalizzazione del capitale relazionale di riferimento ${ }^{26}$, come la loro longa manus. 亡̀ invece possibile ritenere come queste donne siano vittime dei propri legami intimi e familiari all'interno di contesti di devianza normalizzata, piuttosto che autrici di reati. Ogni reale percorso di ricostruzione identitaria non può quindi prescindere dal coinvolgere anche quella fitta rete di persone che costituiscono i legami del "mondo della vita" della stessa donna ammessa alle misure alternative alla detenzione.

criminology in era of backlash", Feminist Criminology, 1(1), 2008, pp. 6-26.

25 Bartholini I., "La riabilitazione extramuraria e il donum/munus del tempo restituito", Studi di sociologia, vol. XII, n. 2, 2014, pag. 154.

26 Banfield E.C., The moral Basis of Blachward Society, The Free Press, Glencoe, 1958. (trad. it. Le basi morali di una società arretrata, il Mulino, Bologna, 2006). 


\begin{tabular}{|c|c|c|c|c|c|c|c|}
\hline \multirow[t]{2}{*}{$\begin{array}{l}\text { REGIONE DI } \\
\text { DETENZIONE }\end{array}$} & \multirow[t]{2}{*}{$\begin{array}{c}\mathrm{N} . \\
\text { ISTITUTI }\end{array}$} & \multirow[t]{2}{*}{$\begin{array}{c}\text { CAPIENZA } \\
\text { REGOLAMENTARE }\end{array}$} & \multicolumn{2}{|c|}{ DETENUti PRESENTI } & \multirow[t]{2}{*}{$\begin{array}{c}\text { DI CUI } \\
\text { STRANIERI }\end{array}$} & \multicolumn{2}{|c|}{$\begin{array}{l}\text { DETENUTI PRESENTI } \\
\text { IN SEMILIBERTÀ }\end{array}$} \\
\hline & & & Tот. & DONNE & & Tот. & STRANIERI \\
\hline ABRUZZO & 8 & 1.502 & 1.817 & 71 & 192 & 13 & 2 \\
\hline BASILICATA & 3 & 470 & 455 & 11 & 65 & 3 & 0 \\
\hline CALABRIA & 13 & 2.662 & 2.397 & 51 & 329 & 19 & 0 \\
\hline CAMPANIA & 17 & 6.082 & 7.188 & 360 & 874 & 195 & 3 \\
\hline EMILIA ROMAGNA & 12 & 2.795 & 2.884 & 117 & 1.347 & 38 & 6 \\
\hline FRIULI-VENEZIA GIULIA & 5 & 484 & 615 & 21 & 231 & 12 & 1 \\
\hline LAZIO & 14 & 5.114 & 5.600 & 390 & 2.417 & 49 & 5 \\
\hline LIGURIA & 7 & 1.174 & 1.411 & 72 & 766 & 27 & 7 \\
\hline LOMBARDIA & 19 & 6.064 & 7.824 & 431 & 3.459 & 55 & 15 \\
\hline MARCHE & 7 & 812 & 869 & 29 & 388 & 9 & 1 \\
\hline MOLISE & 3 & 274 & 322 & $\mathbf{0}$ & 29 & 1 & 0 \\
\hline PIEMONTE & 13 & 3.826 & 3.589 & 126 & 1.551 & 37 & 7 \\
\hline PUGLIA & 11 & 2.377 & 3.280 & 173 & 559 & 78 & 1 \\
\hline SARDEGNA & 12 & 2.774 & 1.839 & 35 & 440 & 18 & 2 \\
\hline SICILIA & 23 & 5.926 & 5.962 & 122 & 1.179 & 86 & 7 \\
\hline TOSCANA & 18 & 3.340 & 3.269 & 113 & 1.573 & 76 & 23 \\
\hline TRENTINO-ALTO ADIGE & 2 & 509 & 289 & 20 & 209 & 2 & 1 \\
\hline UMBRIA & 4 & 1.314 & 1.404 & 42 & 409 & 13 & 0 \\
\hline VALLE D'AOSTA & 1 & 180 & 134 & $\mathbf{0}$ & 84 & 1 & 0 \\
\hline VENETO & 10 & 1.956 & 2.475 & 120 & 1.361 & 38 & 11 \\
\hline TOTALE NAZIONALE & 202 & 49.635 & 53.623 & 2.304 & 17.462 & 770 & 92 \\
\hline
\end{tabular}

Tabella n. 1: Detenuti presenti e capienza regolamentare degli istituti penitenziari per regione di detenzione - Situazione al 31 dicembre 2014(Fonte - Ministero della Giustizia, Dipartimento dell'Amministrazione penitenziaria, Sezione Statistica)

\begin{tabular}{|c|c|}
\hline Affidamento in prova al Servizio sociale & 12.539 \\
\hline Semilibertà & 789 \\
\hline Detenzione domiciliare & 9.635 \\
\hline Lavoro di pubblica utilità & 5.994 \\
\hline Libertà vigilata & 3.588 \\
\hline Libertà controllata & 174 \\
\hline Semidetenzione & 2 \\
\hline Totale generale & 32.721 \\
\hline
\end{tabular}

Tabella n. 2: Misure alternative, lavoro di pubblica utilità, misure di sicurezza, sanzioni sostitutive e messa alla prova - Dati al 30 aprile 2015(Fonte: Ministero Grazia e Giustizia in http://wmw.giustizia.it/giustizialit/mg_1_14_1.wp? facetNode_1=0_2OffacetNode_2=0_2_2oprevisiousPage=mg_1_14\&contentId=SST1145052)

\begin{tabular}{|l|l|}
\hline Numero di dimessi & $10.697(61,5 \%)$ \\
\hline Affidamento in prova in casi particolari & $\mathbf{3 . 4 1 0}(\mathbf{1 9 , 6 \% )}$ \\
\hline Detenzione domiciliare & $3.280(18,9 \%)$ \\
\hline Totale & 17.387 \\
\hline
\end{tabular}

Tabella n. 3: Numero dei dimessi dalla misura alternativa a seguito della misura nel triennio 2012-2014(Fonte: Osservatorio del ministero di Grazia e giustizia [elaborazione nostra]) 


\begin{tabular}{|c|c|}
\hline TIPOLOGIA DELLA MISURA ALTERNATIVA & N. CASI \\
\hline Osservazione detenute (conclusasi con i provvedimenti sotto elencati) & $\mathbf{3 9}$ \\
Detenzione domiciliare & 11 \\
Affidamento in prova al servizio sociale & 3 \\
Affidamento in prova al servizio sociale in casi particolari & 1 \\
Detenzione domiciliare provvisoria & 1 \\
Reclusione in carcere & 23 \\
\hline Detenzione domiciliare (suddivisa in): & $\mathbf{4 4}$ \\
\hline Detenzione domiciliare dalla libertà & 39 \\
\hline Detenzione domiciliare dalla detenzione & 5 \\
\hline Affidamento in prova al servizio sociale (suddivisa in): & $\mathbf{1 7}$ \\
\hline Affidamento in prova al servizio sociale dalla detenzione & 2 \\
\hline Affidamento in prova al servizio sociale dalla libertà & 15 \\
\hline Assistenza familiare & $\mathbf{1}$ \\
\hline Totale dei casi & 101 \\
\hline
\end{tabular}

Tabella n. 4: Distribuzione delle misure alternative disposte tra il 2009-2014 presso l'U.E.P.E. di Agrigento (Fonte - Elaborazione nostra)

\begin{tabular}{|c|c|}
\hline VARIABILE INDAGATA & ESITO DELLA VARIABILE \\
\hline Pena & 50,5 ha ottenuto una pena inferiore ad 1 anno \\
\hline Recidiva & $6,9 \%$ ha reiterato il reato o commesso reati diversi \\
\hline Misure concesse in passato & $95,1 \%$ ha avuto concessa solo una misura alternativa \\
\hline Precedenti penali & $93,1 \%$ non ha commesso reati in passato \\
\hline
\end{tabular}

Tabella n. 5: Esiti delle variabili indagate presso l'U.E.P.E. di Agrigento (Fonte - Elaborazione nostra)

\begin{tabular}{|c|c|}
\hline ABUSIVISMO & $33,6 \%$ \\
\hline REATI CONTRO IL PATRIMONIO & $26,7 \%$ \\
\hline REATI CONTRO LA PERSONA & 19,8 \\
\hline DETENZIONE DI ARMI & $9,9 \%$ \\
\hline OMICIDIO, CONCORSO IN REATO & $9,5 \%$ \\
\hline SFRUTTAMENTO E FAVOREGGIAMENTO DELL'IMMIGRAZIONE & $2,9 \%$ \\
\hline SFRUTTAMENTO E FAVOREGGIAMENTO DELLA PROSTITUZIONE & $1,9 \%$ \\
\hline ALTRI REATI & $6,9 \%$ \\
\hline NON PERVENUTI & $1,9 \%$ \\
\hline
\end{tabular}

Tabella n. 6: Distribuzione dei reati commessi dalle donne del campione preso in esame (Fonte - Elaborazione nostra)

\section{Riferimenti bibliografici.}

- Adler F., Sisters in Crime, McGraw- Hill Book Company New York, 1975.

- Banfield E.C., The moral Basis of Blacbward Society, The Free Press, Glencoe, 1958. (trad. it. Le basi morali di una società arretrata, il Mulino, Bologna, 2006).

- Bartholini I., "Introduzione", in Id. (a cura di), Capitale sociale, reti comunicative e culture di partecipazione, Franco Angeli, Milano, 2008.
- Bartholini I., "La riabilitazione extramuraria e il donum/munus del tempo restituito", Studi di sociologia, vol. XII, n. 2, 2014, p. 151-170.

- Breda R., Coppola C., Sabattini A., Il Servizio Sociale nel sistema penitenziario, Giappichelli Editore, Torino, 1999.

- Buonanno R., L'altra donna. Devianza e criminalità, Adriatica, Bari, 1983.

- Burkhart K.W., Women in Prison, Double-day \& Co, New York, 1976.

- Campelli E., "Una risorsa ambivalente ed incerta: la famiglia", in Campelli E. et 
al. (a cura di), Donne in carcere. Ricerca sulla detenzione femminile in Italia, Feltrinelli, Milano, 1992.

- Campelli E., Faccioli F., Giordano V., Pitch T., Donne in carcere. Ricerca sulla detenzione femminile in Italia, Feltrinelli, Milano, 1992.

- Carlen P., Worrall A., Analysing women's imprisonment, Willan, Cullompton, 2004.

- Chesney-Lind M., Irwin K., "Patriarchy, crime and justice: Feminist criminology in era of backlash", Feminist Criminology, 1(1), 2008, pp. 6-26.

- de Cataldo Neuburger L., La criminalità femminile tra stereotipi culturali e malintese realtà, Cedam, Padova, 1996.

- Di Cara M., Gervasoni A., Steiner M.A., Riforma penitenziaria e intervento sociale, La Nuova Italia Scientifica, Roma, 1990.

- Dino A. (in collaborazione con Principato T.), Mafia Donna. Le vestali del sacro e dell'onore, Flaccovio, Palermo, 1997.

- Dino A., Mutazioni. Etnografia del mondo di Cosa Nostra, La Zisa, Palermo, 2002.

- Dino A., Gli ultimi padrini. Indagine sul governo di Cosa Nostra, Laterza, RomaBari, 2011.

- Edwards S., Gender, sex and law, Croom Helm, London, 1985.

- Faccioli F., I soggetti deboli. I giovani e le donne nel sistema penale, Franco Angeli, Milano, 1990.

- Faccioli F., "Le donne in carcere: la composizione sociale, i reati, le pene", in Campelli E. et al., Donne in carcere. Ricerca sulla detenzione femminile in Italia, Feltrinelli, Milano, 1992.

- Feinman C., The Criminalization of a Woman's Body, Routledge, London, 1992.

- Frudà L., Alternative al carcere. Percorsi, attori e reti sociali nell'esecurione penale esterna: un approfondimento della ricerca applicata, Franco Angeli, Milano, 2006.

- Gelsthorpe L., "Female offending': a theoretical overview", in McIvor G. (ed.), "Women who offend". Research Highlights in Social Work 44, Jessica Kingsley Publishers, London, 2004.

- Gucciardo G., "Familismo amorale, modelli culturali e azione razionale", in Bartholini I. (a cura di), Capitale sociale, reti comunicative e culture di partecipazione, Franco Angeli, Milano, 2008.
- Hagan J., Crime and Disrepute. Sociology of new Century, Pine Forge Press, Thousand Oaks (California), 1994.

- Hedderman C., Empty cells or empty words, Criminal Justice Alliance, London, 2012.

- Leonardi F., "Le misure alternative alla detenzione tra reinserimento sociale e abbattimento della recidiva", Rassegna di Studi penitenziari, n. 2, 2007, pp. 7-26.

- Minicucci G., Qui e altrove. Famiglie di Calabria e Argentina, Franco Angeli, Milano, 1989.

- Mosconi G., Sarzotti C., Antigone in carcere, Carocci Editore, Roma, 2004.

- Naffine N., Feminism and Criminology, Cambridge Polity Press, Cambridge, 1997.

- Patel S., Stanley S., The use of the community order and the suspended sentence order for women, Center for Crime and Justice Studies, London, 2008.

- Pelanda D., Mondo recluso. Vivere in carcere in Italia oggi, Effatà, Torino, 2010.

- Pitch T., Donne in carcere. Ricerca sulla detenzione femminile in Italia, Feltrinelli, Milano, 1992.

- Pitch T., "Dove si vive, come si vive", in Campelli E., Faccioli F., Giordano V., Pitch T., Donne in carcere. Ricerca sulla detenzione femminile in Italia, Feltrinelli, Milano, 1992.

- Santoro E., Carcere e società liberale, Torino, Giappichelli, 2004.

- Sibert R., Le donne, la mafia, Il Saggiatore, Milano, 1994.

- Sibert R., Mafia e quotidianità, Il Saggiatore, Milano, 1996.

- Thomas C.W., "Prisonization or Resocialization? A Study of External Factors Associated with the Impact of Imprisonment", Journal of research in crime and Delinquency, 10(13), 1973, pp.13-21.

- Torrente G. "Indulto. La verità, tutta la verità, nient'altro che la verità", Innocenti evasioni. La privazione della libertà e le libertà possibili, reperibile on line: http://www.innocentievasioni.net/inde x.php?option $=$ com_content\&view $=$ arti cle\&id $=71$

- Vitello F., Stefanizzi S., "Genere e criminalità: alcune riflessioni sui dati delle statistiche giudiziarie", in de Cataldo Neuburger L., La criminalità 
femminile tra stereotipi culturali e malintese realtà, Cedam, Padova, 1996.

- Walker H., “Women's issues in probation practice", in H. Walker \& B.
Beamount (eds.), Working with offenders, Macmillan, London, 1985.

- Zucca M., Donne delinquenti. Storie di streghe, eretiche, ribelli, rivoltose, tarantolate, Edizioni Simone, Napoli, 2004. 